\title{
AN EXTENSION OF ASCOLI'S THEOREM AND ITS APPLICATIONS TO THE THEORY OF OPTIMAL CONTROL
}

\author{
BY \\ S. S. L. CHANG
}

I. Introduction. Ascoli's theorem deals with continuous functions and states that the space of bounded, equicontinuous functions is compact. The present paper extends it to the measurable functions. The space of bounded "equimeasurable functions," is compact, and it contains the bounded equicontinuous functions as a subset.

The above theorem is applied to two problems in the theory of optimal control:

1. To give an existence proof of optimal control among allowed control functions which are measurable and enter the system equations in a nonlinear manner.

2. To derive a necessary condition for optimal control in bounded phase space (Theorem 8). The condition is different and simpler than the one derived previously by Gamkrelidze [1]. It is proved to be also sufficient for linear systems, and its applications to engineering problems are given in previous papers [2].

In their classic paper [3], Boltjanskiĭ, Gamkrelidze, and Pontryagin derived the "maximum principle" by assuming the existence of optimal control. Gamkrelidze [4] gave an existence proof for the linear case with discontinuous control. Markus and Lee [5] sketched an existence proof for the linear case with discontinuous control and also stated an existence theorem for the nonlinear case with continuous control satisfying a Lipschitz condition. None of these existence proofs are sufficiently general to form a basis for proving Pontryagin's maximum principle and the above-mentioned condition for optimal control in bounded phase space.

The method used in deriving the latter is also different from that used by earlier investigators. In place of the rigid bound, a cost function with a multiplier $K$ is introduced for regions beyond the boundaries in phase space. It is shown that in the limit of the multiplier $K$ approaching infinity, both the added cost and the maximum excursion of the optimal path beyond the boundaries approach zero, and the condition for optimal control is thus derived.

The generalized Ascoli theorem is useful in both the existence proof of optimal control, and in proving the existence of a limit as $K$ approaches infinity.

\footnotetext{
Presented to the Society, January 24, 1964; received by the editors September 7, 1961 and, in revised form, July 18, 1963.
} 


\section{Equimeasurable functions.}

Definition. A function $f(t)$ is said to be measurable if there exists a sequence of step functions $g_{k}(t)$ such that

$$
\lim _{k \rightarrow \infty}\left|g_{k}(t)-f(t)\right| \rightarrow 0
$$

except on a set of zero measure.

An infinite set of functions $F$ is said to be equimeasurable on a closed interval $T$ if there exists a sequence of step functions $g_{i k}(t)$ for each $f_{i}(t) \in F$ such that the following conditions are satisfied:

2.1. $g_{i k}(t)$ has no more than $k$ discontinuities.

2.2. For every $\epsilon_{1}>0$ and $\delta_{1}>0$, there is a finite number $K\left(\epsilon_{1}, \delta_{1}\right)$ and for every $g_{i k}(t)$ with $k>K\left(\epsilon_{1}, \delta_{1}\right)$

$$
\left|g_{i k}(t)-f_{i}(t)\right|<\epsilon_{1}, \quad i=1,2, \cdots, \infty,
$$

except on a set of total measure less than $\delta_{1}$.

THEOREM 1. The following sets of functions are equimeasurable:

(a) functions with uniformly bounded variations on $T$ (same bounds for all the functions belonging to the set),

(b) piecewise equicontinuous functions,

(c) functions obtained by a finite number of addition, subtraction, and multiplication operations on equimeasurable functions.

Proof. (a) Given a function $f(t)$ with bounded variation $M$ in an interval $T=\left[t_{1}, t_{2}\right]$, and $K\left(\epsilon_{1}, \delta_{1}\right)$ is selected to be

$$
K\left(\epsilon_{1}, \delta_{1}\right) \equiv \frac{M\left(t_{2}-t_{1}\right)}{2 \epsilon_{1} \delta_{1}} .
$$

The interval $T$ is divided into $k$ subintervals $\tau_{i}$ of equal length. Let

$$
a_{i}=\sup _{t \in \tau_{i}} f(t), \quad b_{i}=\inf _{t \in \tau_{i}} f(t) .
$$

The step function $g_{k}(t)$ is defined by

$$
g_{k}(t) \equiv \frac{1}{2}\left(a_{i}+b_{i}\right), \quad t \in \tau_{i}
$$

Since the maximum variation is $M$, the number of intervals with $a_{i}-b_{i}$ $\geqq 2 \epsilon_{1}$ is not greater than $M / 2 \epsilon_{1}$. Therefore, for any $k>K\left(\epsilon_{1}, \delta_{1}\right)$

$$
\left|f(t)-g_{k}(t)\right|<\epsilon_{1}
$$

with the exception of at most $M / 2 \epsilon_{1}$ intervals, the total length of which is

$$
\frac{M}{2 \epsilon_{1}} \frac{\left(t_{1}-t_{2}\right)}{k}<\delta_{1} \text {. }
$$

(b) For piecewise equicontinuous functions, as $T$ is bounded and closed, 
a modulus of continuity $\theta(\Delta)$ can be defined [6] which holds for all functions $f_{i}$ and $b \in T$ :

$$
\left|f_{i}(b+\Delta)-f_{i}(b)\right|<\theta(\Delta) .
$$

$\theta(\Delta)=\theta(|\Delta|) \rightarrow 0$ as $|\Delta| \rightarrow 0$ except at a finite number $(<N)$ of discontinuities which can be different for different $f_{i}$. Given any $\epsilon_{1}, \delta_{1}$, a $\Delta_{1}$ is defined as the smaller of the two, $\Delta_{11}$ and $\Delta_{12}$ :

$$
\theta\left(\Delta_{11}\right)=\epsilon_{1}, \quad \Delta_{12}=\frac{\delta_{1}}{2 N} .
$$

Then

$$
K\left(\epsilon_{1}, \delta_{1}\right)=\frac{t_{2}-t_{1}}{\Delta_{1}}
$$

For each value of $k$, the interval $T$ is divided into $k$ equal intervals and $g_{i k}(t)$ is assigned the mean value of $f_{i}(t)$ in each interval. Then (1) is satisfied. Q.E.D.

In the following, the functions $f_{i}(t)$ are assumed to be bounded from above and below on an interval $T$. Consequently the step functions $g_{i k}(t)$ are also bounded from above and below. Let $S_{i}$ denote the sequence of step functions $g_{i k}(t), k=1,2, \cdots, \infty$. Let $G_{k}$ denote the sequence of step functions $g_{i k}(t)$, $i=1,2, \cdots, \infty$. Let $L$ denote the length of the interval $T$. In what follows, the length and measure of a subset $T^{\prime}$ of $T$ are synonymous, and is denoted by $\mu\left\{T^{\prime}\right\}$.

LEMma 1. Given any $k, a$ sequence of functions $\mathbf{g}_{n k}(t)$ can be selected from $G_{k}$ such that it converges in measure to some function $V_{k}(t)$ :

$$
\mu\left\{t|| \mathbf{g}_{n k}-V_{k} \mid \geqq \frac{1}{n}\right\}<\frac{L}{n}
$$

Furthermore, each $\mathbf{g}_{n k}(t)$ is selected from the subset of $G_{k}$ with $i \geqq n$.

Proof. Let $t_{i 1}, t_{i 2}, \cdots, t_{i k^{\prime}}$ denote all the points of discontinuity of $g_{i k}(t)$ and $t_{i\left(k^{\prime}+1\right)} \cdots t_{i k}$ be any other points in $T$. These $k$ points partition the interval $T$ into $k+1$ subintervals. Let $a_{i 1}, a_{i 2}, \cdots, a_{i(k+1)}$ be the values of $g_{i k}(t)$ in the subintervals. The set $t_{i 1}, t_{i 2}, \cdots, t_{i k}, a_{i 1}, a_{i 2}, \cdots, a_{i(k+1)}$ defines a point $P_{i}$ in a bounded $(2 k+1)$-dimensional space. It follows from the Weierstrass-Bolzano theorem that the set of points $\left\{P_{i}\right\}$ representing $G_{k}$ has at least one point of accumulation $t_{1}, t_{2}, \cdots, t_{k}, a_{1}, a_{2}, \cdots, a_{k+1}$. Therefore, a sequence of points $\left\{P_{(n)}\right\}$ can be so selected that

$$
\begin{aligned}
\left|t_{(n) l}-t_{l}\right| & \leqq \frac{L}{n k}, \quad l=1,2, \cdots, k, \\
\left|a_{(n) l}-a_{l}\right| \leqq \frac{1}{n}, & l=1,2, \cdots, k+1 .
\end{aligned}
$$


The step function specified by $t_{1}, t_{2}, \cdots, t_{k}, a_{1}, a_{2}, \cdots, a_{k+1}$ is $V_{k}(t)$, and $P_{(n)}$ is the representative point of $\mathbf{g}_{n k}(t)$ in (2).

The condition $i \geqq n$ can be met as follows: After selecting each $P_{\left(n^{\prime}\right)}$ which is the representative point of $g_{i^{\prime} k}(t)$, the next point $P_{\left(n^{\prime}+1\right)}$ is selected from the subset $\left\{P_{i^{\prime \prime}}\right\}$ with $i^{\prime \prime}>i^{\prime}$. Q.E.D.

Selection of two-dimensional array $\left\{\left\{\mathbf{g}_{n k}(t)\right\}\right\}$. Let the sequence $\left\{\mathbf{g}_{n k}(t)\right\}$ be denoted $H_{k}$. Then the sequence $H_{k+1}$ is selected from $G_{k+1}$ as follows.

Each term $g_{n k}(t)$ in $H_{k}$ is identical with a $g_{i k}(t)$ in $G_{k}$. Correspondingly there is a $g_{i(k+1)}(t)$ in $G_{k+1}$, and all the $g_{i(k+1)}(t)$ so selected constitute a subset $G_{k+1}^{\prime}$ of $G_{k+1}$. The sequence $\mathbf{g}_{n(k+1)}(t)$ is selected from $G_{k+1}^{\prime}$ in exactly the same way as $\mathbf{g}_{n k}(t)$ is selected from $G_{k}$. In identical manner $\mathbf{g}_{n(k+2)}$ is selected from the subset $G_{k+2}^{\prime}$ of $G_{k+2}$, etc.

Using the above procedure, and starting from $k=1$, one obtains the twodimensional array of functions $\left\{\left\{\mathrm{g}_{n k}(t)\right\}\right\}, k=1,2, \cdots, \infty$ and $n=1,2, \cdots, \infty$. Each member of the array satisfies (2) and the following condition:

For any $\mathbf{g}_{n k}(t)$, and $k^{\prime}<k$, there is a $\mathbf{g}_{n^{\prime} k^{\prime}}(t)$ which belongs to the same sequence $S_{i}$. That is,

$$
\mathbf{g}_{n k}(t) \equiv g_{i k}(t)
$$

and

$$
\mathbf{g}_{n^{\prime} k^{\prime}}(t) \equiv g_{i k^{\prime}}(t) \quad i \geqq n^{\prime} \geqq n .
$$

Lemma 2. The diagonal sequence $\left\{\mathbf{g}_{n n}(t)\right\}$ of the array $\left\{\left\{\mathbf{g}_{n k}(t)\right\}\right\}$ is a Cauchy sequence in measure.

Proof. Given $\epsilon$ and $\delta$, there is a $K(\epsilon / 4, \delta / 4)$ as defined by (1). Let $I(\epsilon, \delta)$ denote the least integer which is larger than all three: $K(\epsilon / 4, \delta / 4), 4 / \epsilon$ and $4 L / \delta$. Consider any two functions in the sequence $\mathrm{g}_{n n}(t)$ and $\mathbf{g}_{m m}(t)$ with $n>m>I(\epsilon, \delta)$. Because of the rule of selection, there is a $\mathbf{g}_{s m}(t)$ with $s \geqq n$ which belongs to the same $S_{i}$ with $\mathrm{g}_{n n}(t)$.

Inequality (1) gives

$$
\left|\mathbf{g}_{n n}(t)-\mathbf{g}_{s m}(t)\right| \leqq\left|\mathbf{g}_{n n}(t)-f_{i}(t)\right|+\left|\mathbf{g}_{s m}(t)-f_{i}(t)\right| \leqq \frac{\epsilon}{2}
$$

except on a set of total measure less than $\delta / 2$. Inequality (2) gives

$$
\begin{aligned}
\left|\mathbf{g}_{s m}(t)-\mathbf{g}_{m m}(t)\right| & \leqq\left|\mathbf{g}_{s m}(t)-V_{m}(t)\right|+\left|\mathbf{g}_{n m}(t)-V_{n}(t)\right| \\
& \leqq \frac{1}{s}+\frac{1}{m}<\frac{\epsilon}{2} .
\end{aligned}
$$

Combining (3) and (4) gives

$$
\mu\left\{t|| \mathbf{g}_{n n}-\mathbf{g}_{m m} \mid \geqq \epsilon\right\}<\delta .
$$

Q.E.D. 
A by-product of the above proof is that no two members of the sequence $\left\{\mathbf{g}_{n n}\right\}$ belong to the same $S_{i}$. For each $\mathbf{g}_{n^{\prime} n^{\prime}}(t)$ there corresponds an $S_{i^{\prime}}$ and $f_{i^{\prime}}(t)$. Let $f_{i^{\prime}}(t)$ be redenoted $f_{n^{\prime}}(t)$. Then all members of the sequence $\left\{f_{n}(t)\right\}$, $n=1,2, \cdots, \infty$, are distinct. It follows from equations (1) and (5) that $\left\{f_{n}(t)\right\}$ is a Cauchy sequence in measure.

THeOREm 2. Let $\left\{f_{i}(t)\right\}$ be an equimeasurable set of functions which are bounded from above and below. An infinite subsequence $\left\{f_{(n)}(t)\right\}$ can be selected from $\left\{f_{i}(t)\right\}$ such that $f_{(n)}(t)$ converges almost uniformly to a measurable function $v(t)$.

Proof. By Lemma 2, a Cauchy sequence in measure $\left\{f_{n}(t)\right\}$ is selected from $\left\{f_{i}(t)\right\}$. A subsequence of $\left\{f_{n}(t)\right\}$ is a Cauchy sequence [almost uniform] [7]. Let it be denoted by $\left\{\mid f_{(n)}(t)\right\}$. By Cauchy's theorem $[8],\left\{f_{(n)}(t)\right\}$ converges to a measurable function almost uniformly on T. Q.E.D.

Definitions. The norm of a vector $\alpha$ is defined as

$$
\left\|\left(\alpha_{1}, \alpha_{2}, \cdots, \alpha_{N}\right)\right\|=\sup _{i=1,2, \cdots, N}\left|\alpha_{i}\right| .
$$

A vector function has a finite number of components. A set of vector functions $\left\{\boldsymbol{w}_{i}(t)\right\}$ is said to be equimeasurable if conditions 2.1 and 2.2 are valid with (1) replaced by

$$
\boldsymbol{\mu}\left\{t \mid\left\|\boldsymbol{g}_{i k}-\boldsymbol{w}_{i}\right\| \geqq \epsilon_{1}\right\}<\delta_{1} .
$$

The norm of vector functions takes the place of the absolute values of scalar functions.

Corollary 1. An infinite subsequence $\left\{\boldsymbol{w}_{(n)}(t)\right\}$ can be selected from an equimeasurable set $\left\{\boldsymbol{w}_{i}(t)\right\}$ such that $\boldsymbol{w}_{(n)}(t)$ converges almost uniformly to a measurable function $V(t)$.

Proof. Let $w_{i}^{j}(t)$ denote the $j$ th component of $w_{i}(t)$. From each vector function $w_{i}(t)$, a scalar function $f_{i}(t)$ can be defined:

$$
f_{i}(t+(j-1) L)=w_{i}^{\prime}(t), \quad j=1,2, \cdots, N .
$$

Then $\left\{f_{i}(t)\right\}$ is an equimeasurable set defined on an interval of length $N L$. By Theorem 2, a subsequence $\left\{f_{(n)}(t)\right\}$ can be selected from $\left\{f_{i}(t)\right\}$ such that it converges almost uniformly to $U(t)$. By decomposing $f_{(n)}(t)$ and $U(t), w_{(n)}(t)$ and $V(t)$ are obtained. Q.E.D.

CoRollary 2. Let $B$ denote an enumerably infinite set of vector functions $\{w(t)\}$. Each $w(t)$ has $m$ components $w^{1}(t), w^{2}(t), \cdots, w^{m}(t)$ which are uniformly bounded on a closed interval $T$. The components $w^{\mu}(t), 1 \leqq \mu \leqq m_{1}$, are equicontinuous; the components $w^{\nu}(t), m_{1}+1 \leqq \nu \leqq m$, are equimeasurable.

Then from $B$ a subset of vector functions $\left\{w_{(n)}(t)\right\}, n=1,2, \cdots, \infty$, can be 
selected which converges to a limit $V(t)$ in the sense that the components $W_{(n)}^{u}(t)$ converge uniformly to $V^{\mu}(t)$ and the components $W_{(n)}^{\nu}(t)$ converge almost uniformly to $V^{2}(t)$.

Proof. As equicontinuous functions are equimeasurable, by Corollary 1, a subsequence $\left\{W_{(n)}(t)\right\}$ can be selected which converges uniformly to $V(t)$ except on a set $T^{\prime}$ of zero measure. Then $V^{\mu}(t)$ for $t \in T^{\prime}, \mu=1,2, \cdots, m_{1}$ can be defined as the limit of $V^{\mu}(t), t \in T-T^{\prime}$, from either side. The uniform convergence of $\left\{W_{(n)}^{u}(t)\right\}$ to $V^{\mu}(t)$ on $T^{\prime}$ follows from Moore's theorem [9]. Q.E.D.

III. The control problem. The controlled system is described by a set of first order differential equations

$$
\dot{x}^{i}=f^{i}(x, u, t), \quad i=0,1,2, \cdots, n_{1},
$$

where $x$ and $u$ stand for the two vectors $\left(x^{1}, x^{2}, \cdots, x^{n_{1}}\right)$, and $\left(u^{1}, u^{2}, \cdots, u^{n_{2}}\right)$, respectively, and $\mathrm{x}$ will be used to note the vector $\left(x^{0}, x^{1}, \cdots, x^{n_{1}}\right)$. The functions $f^{i}$ together with partial derivatives $\partial f^{i} / \partial x^{j}, i, j=0,1,2, \ldots, n_{1}$ are single valued, bounded, and continuous in all the variables $x, u$, and $t$ on a product region $X_{1} U_{1} T_{1}$ where $X_{1}, U_{1}$ are open regions in the $n_{1}$-dimensional $x$-space and $n_{2}$-dimensional $u$-space, and $T_{1}$ is an open interval of $t$, (see Note 1). It is understood that all the properties and relationships stated in the subsequent sections are on the $X_{1} U_{1} T_{1}$.

Note 1. The existence of bounded and continuous derivatives in $x$ is required for proving Pontryagin's maximum principle, but not required for proving the existence theorems. For the latter, it is sufficient to assume that $\mathrm{f}(\boldsymbol{x}, \boldsymbol{u}, t)$ satisfies Lipschitz's condition in $\boldsymbol{x}$.

It follows from the Caratheodory existence theorem that if the initial $\boldsymbol{x}\left(t_{1}\right)$ and $\boldsymbol{u}(t)$ for a subsequent interval are given, $\boldsymbol{x}(t)$ is uniquely determined on the same interval. Thus an allowed control can be defined as a function $u(t)$ satisfying the following conditions:

3.1.

$$
u(t) \in U, \quad t_{1} \leqq t \leqq t_{2}
$$

where $U$ is a closed bounded region in $U_{1}$. The closed interval $\left[t_{1}, t_{2}\right]$ is denoted by $T, T \in T_{1}$.

3.2. There is a finite $K(\epsilon, \delta)$ for each $\epsilon>0$ and $\delta>0$, and $K(\epsilon, \delta)$ is the same for all $\boldsymbol{u}(t)$. A sequence of step functions $\boldsymbol{g}_{k}(t)$ can be found for $\boldsymbol{u}(t)$ such that for each $k \geqq K(\epsilon, \delta)$ :

$$
\mu\left\{T \mid\left\|\boldsymbol{g}_{k}(t)-\boldsymbol{u}(t)\right\| \geqq \epsilon\right\}<\delta .
$$

3.3. The $\boldsymbol{x}(t)$ resulting from $\boldsymbol{u}(t)$ satisfies

$$
\boldsymbol{x}(t) \in X, \quad t_{1} \leqq t \leqq t_{2},
$$


where $X$ is a closed region in $X_{1}$. Note that in the special case of $X$ being the entire accessible region (accessible under the constraint that (9) and 3.2 are satisfied by $\boldsymbol{u}(t)$, the problem is reduced to one without bound in $\boldsymbol{x}$-space. The function $\boldsymbol{x}(t)$ resulting from an allowed control $u(t)$ is called an allowed path.

In the following sections the following distinction will be made: $x, u$ imply $\boldsymbol{x}(t), \boldsymbol{u}(t)$ for the entire path, $t_{1}<t<t_{2} ; \boldsymbol{x}\left(t_{3}\right), \boldsymbol{u}\left(t_{3}\right)$, etc., denote the values at a particular instant $t_{3}$.

The set of all allowed controls is denoted by $C$. The set of all allowed paths is denoted by $P$. The set of all allowed control-and-path pairs $u, x$ is denoted by $A$.

A return function or functional $R$ is defined in terms of the $x^{0}\left(t_{2}\right)$ resulting from $u$

$$
R[u]=x^{0}\left(t_{2}\right)-x^{0}\left(t_{1}\right) .
$$

There are two basic types of terminating conditions: (a) $t_{2}$ is fixed, and (b) $x\left(t_{2}\right)$ is fixed, and $t_{1} \leqq t_{2} \leqq T_{2}$ but $t_{2}$ is otherwise unknown. The interval $\left[t_{1}, T_{2}\right] \subset T_{1}$. The condition (a) is referred to as "free end point." The condition (b) is referred to as "fixed end point."

An allowed control $u_{1} \in C$ is said to be optimal if

$$
R\left[u_{1}\right]=\sup _{u \in C} R(u) .
$$

In any given problem, there may be more than one optimal control. The set of optimal controls under free end point conditions is denoted as $S_{0}$, and the set of optimal controls under fixed end point conditions is denoted as $S_{0}\left(T_{2}\right)$.

Theorem 3. $A$ is compact.

Proof. Let $u, \mathrm{x}$ be an allowed control and path pair. An $\left(n_{1}+n_{2}+1\right)$ dimensional vector function $w(t)$ is formulated from the components of $\mathbf{x}(t)$ and $\boldsymbol{u}(t): w^{i}(t)=x^{i}(t), i=0,1, \cdots, n_{1} ; w^{j+n_{1}}(t)=u^{j}(t), \quad j=1,2, \cdots, n_{2}$. Let $R$ be a set of $w(t)$ with infinite members.

The components $x$ are bounded and equicontinuous because $f(x, u, t)$ is bounded and $t_{2}-t_{1}$ is finite. The components $u$ are bounded and equimeasurable by definition. Corollary 2 of Theorem 2 states that a sequence $\boldsymbol{w}_{(k)}(t)$ can be selected from $B$ which converges to a limit function $V(t), V(t)$ $=\left(V^{0}, V^{1}, \cdots, V^{n_{1}+n_{2}}\right)$. Because $X$ is closed, and the vector $\left(w_{(k)}^{1}, w_{(k)}^{2}, \cdots\right.$, $\left.w_{(k)}^{n_{1}}\right) \in X$ for $k=1,2, \cdots, \infty,\left(V^{1}, V^{2}, \cdots, V^{n_{1}}\right) \in X$. Similarly, $\left(V^{n_{1}+1}, \cdots\right.$, $\left.V^{n_{1}+n_{2}}\right) \in U$ almost everywhere except on a subset $T_{L}^{\prime}$ of zero measure. Let $\hat{u}(t)$ be defined as

$$
\hat{u}^{i}(t)=V^{n_{1}+i}(t), \quad t \in T-T_{L}^{\prime}
$$

and $\hat{u}(t)$ be any vector $\in U$, on the subset $T_{L}^{\prime}$. Then $\hat{u}(t) \in U$ on $T$. 
Let $\hat{\mathbf{x}}(t)$ denote the vector $\left(V^{0}(t), V^{1}(t), \cdots, V^{n_{1}}(t)\right)$. It remains to be shown that (8) is satisfied by $(\hat{\mathbf{x}}(t), \hat{u}(t))$. If

$$
\left\|\hat{\mathbf{x}}\left(t_{3}\right)-\int_{t_{1}}^{t_{3}} \mathbf{f}(\hat{x}, \hat{u}, t) d t-\mathbf{x}\left(t_{1}\right)\right\|>a>0
$$

for any $t_{3} \in T$, then a contradiction can be shown as follows:

Since $\mathrm{f}(x, u, t)$ is continuous in $x$ and $u$ on a closed set, it is uniformly continuous [10]. For any $\epsilon_{2}>0$, it is possible to find a $\delta_{2}\left(\epsilon_{2}\right)>0$ such that

$$
\|\mathrm{f}(\boldsymbol{x}, \boldsymbol{u}, t)-\mathrm{f}(\hat{\boldsymbol{x}}, \hat{u}, t)\|<\epsilon_{2}
$$

for all $x$ and $u$ satisfying

$$
\|x-\hat{x}\|<\delta_{2}\left(\epsilon_{2}\right)
$$

and

$$
\|u-\hat{u}\|<\delta_{2}\left(\epsilon_{2}\right)
$$

Let

$$
\begin{aligned}
& \epsilon_{2}=\frac{a}{3\left(t_{2}-t_{1}\right)}, \\
& \epsilon_{3}=\inf \left\{\frac{a}{3}, \delta_{2}\left(\epsilon_{2}\right)\right\}, \\
& \beta_{f} \equiv \sup _{x, u, t \in X, U, T}\|\mathrm{f}(x, u, t)\|, \\
& \delta_{3}=\frac{a}{6 \beta_{f}} .
\end{aligned}
$$

For sufficiently large $k$, the components $x_{(k)}(t)$ and $u_{(k)}(t)$ of $w_{(k)}(t)$ satisfy the following inequalities:

$$
\begin{gathered}
\left\|\mathrm{x}_{(k)}(t)-\hat{\mathbf{x}}(t)\right\|<\epsilon_{3}<\frac{a}{3}, \\
\left\|\int_{t_{1}}^{t_{3}} \mathbf{f}\left(\boldsymbol{x}_{(k)}, u_{(k)}, t\right) d t-\int_{t_{1}}^{t_{3}} f(\hat{x}, \hat{u}, t) d t\right\| \\
<\delta_{3} \cdot 2 \beta_{f}+\epsilon_{2}\left(t_{3}-t_{1}\right) \leqq \frac{2 a}{3} .
\end{gathered}
$$

As $u_{(k)}$ and $\mathbf{x}_{(k)}$ constitute an allowed control and path pair

$$
\mathbf{x}_{(k)}\left(t_{3}\right)=\int_{t_{1}}^{t_{3}} f\left(x_{(k)}, u_{(k)}, t\right) d t+\mathbf{x}\left(t_{1}\right) .
$$

The inequality (14) is contradicted by (15), (16), and (17). Therefore 


$$
\hat{\mathbf{x}}\left(t_{3}\right)=\int_{t_{1}}^{t_{3}} \mathbf{f}(\hat{\mathbf{x}}, \hat{u}, t) d t+\mathbf{x}\left(t_{1}\right)
$$

Differentiating the above equation with respect to $t_{3}$ and then setting $t_{3}=t$, (8) is obtained for $\hat{\mathbf{x}}$ and $\hat{u}$. Q.E.D.

\section{Existence theorems.}

Definitions. Let the set of all allowed paths $x$ satisfying $\mathbf{x}\left(t_{1}\right)=\xi_{1}$ be denoted by $p\left(t_{1}, \xi_{1}\right)$. The set $\mathbf{x}\left(t_{3}\right)$, with $t_{3} \geqq t_{1}$ and $\mathbf{x} \in p\left(t_{1}, \xi_{1}\right)$, is denoted by $\Omega\left(t_{3}\right)$. It is the accessible region at $t_{3}$ with the initial condition understood. The set of points $\mathrm{x}(t)$, with $t_{1} \leqq t \leqq t_{3}$ and $\mathrm{x} \in p\left(t_{1}, \xi_{1}\right)$, is denoted by $\Omega\left(t \leqq t_{3}\right)$. It is the accessible region up to and including $t_{3}$.

The distance between a point $\mathrm{x}$ and a region $R$ is defined as

$$
d(\mathbf{x}, R) \equiv \inf _{x^{\prime} \in R}\left\|\mathbf{x}-\mathbf{x}^{\prime}\right\|
$$

The set of $\mathrm{x}$ with $d(\mathrm{x}, R)<\epsilon$ is called the $\epsilon$-neighborhood of $R$, or simply $N_{\epsilon}(R)$.

A boundary point $\xi$ of $X$ is called returnable if there is a $u(t) \in U$ such that $f(\xi, u, t)$ points inward (of $X)$. Consequently, if $x(t)=\xi$, the path $x(t)$ can be continued to some larger value of $t$.

THEOREM 4. $\Omega\left(t_{3}\right)$ is closed.

Proof. Let $\mathrm{y}$ be the limit point of a sequence $\mathrm{y}_{(k)}$, and each $\mathrm{y}_{(k)} \in \Omega\left(t_{3}\right)$. By definition of $\Omega\left(t_{3}\right)$, there is an allowed path $\mathbf{x}_{(k)} \in p\left(t_{1}, \xi_{1}\right)$ that $\mathbf{x}_{(k)}\left(t_{3}\right)=\mathbf{y}_{(k)}$.

Theorem 3 states that a subsequence can be selected from $\left\{\mathbf{x}_{(k)}\right\}$ to converge on an allowed path $x$. Therefore, there is a subsequence in $y_{(k)}$ which converges to a limit $\hat{\mathbf{x}}\left(t_{3}\right)$. Therefore, $y=\hat{x}\left(t_{3}\right) . y \in \Omega\left(t_{3}\right)$. Q.E.D.

Lemma 3. If $\mathbf{x}\left(t_{3}\right)$ of an allowed path is either an interior point or a returnable boundary point of $X$, then given $\epsilon>0$, there is a $\tau(\epsilon)$ such that $\mathbf{x}\left(t_{3}\right)$ is in the $\epsilon$-neighborhood of $\Omega(t)$ for every $t$ satisfying $\left|t-t_{3}\right|<\tau(\epsilon)$.

Proof. Lemma 3 is due to the boundedness of f. For sufficiently small $\epsilon$, $\tau(\epsilon)=\epsilon / \beta_{f}$. Q.E.D.

THEOREM 5. If all the common boundary points of $\Omega\left(t \leqq t_{3}\right)$ and $X$ belong to the returnable set, then $\Omega\left(t \leqq t_{3}\right)$ is closed.

Proof. Let $\mathbf{y}$ be the limit point of a sequence $\mathbf{y}_{(k)}$, and each $\mathbf{y}_{(k)} \in \Omega\left(t \leqq t_{3}\right)$. Then one can find a set $t_{(k)}$ so that $\mathrm{y}_{(k)} \in \Omega\left(t_{(k)}\right)$ for all $k$. Since there are infinite $t_{(k)}$ in the closed interval $\left[t_{1}, t_{3}\right]$, there is at least one point of accumulation. Let it be denoted $\hat{t}$. Then a subsequence $\mathrm{y}_{[k]}$ can be selected from $\mathbf{y}_{(k)}$ so that

$$
\lim y_{[k]} \rightarrow y, \quad \lim t_{[k]} \rightarrow t
$$


Since $\left[t_{1}, t_{3}\right]$ is closed, $t \in\left[t_{1}, t_{3}\right]$, and $\Omega(t) \subset \Omega\left(t \leqq t_{3}\right)$. Also $\Omega(t)$ is closed because of Theorem 4 .

If $y \notin \Omega(t)$, an $\epsilon_{1}>0$ can be found such that

$$
d(\mathrm{y}, \Omega(t))>\epsilon_{1} \text {. }
$$

For sufficiently large $k$

$$
\begin{aligned}
\mathbf{y}_{[k]}-\mathbf{y} & <\frac{\epsilon_{1}}{2}, \\
\imath-t_{[k]} & <\tau\left(\frac{\epsilon_{1}}{2}\right) .
\end{aligned}
$$

Lemma 3 gives

$$
d\left(\mathrm{y}_{|k|}, \Omega(t)\right)<\frac{\epsilon_{1}}{2}
$$

Inequalities (20) and (21) contradict (19). Q.E.D.

TheOREM 6. If there is an allowed control, there is an optimal control with free end point. ( $S_{0}$ is not empty.)

Proof. By definition (12) of $R(u)$, the problem is whether there is an $\mathrm{x} \in P$ such that

$$
x^{0}\left(t_{2}\right)=M \equiv \sup _{x \in P} x^{0}\left(t_{2}\right) .
$$

If $M$ is not equal to one of the $x^{0}\left(t_{2}\right)$ in the set, there is a sequence $\mathbf{x}_{(n)} \in P$ such that

$$
\lim x_{(n)}^{0}\left(t_{2}\right) \rightarrow M
$$

By Theorem 3, a subsequence $x_{\{n\}}$ can be selected to converge to $\hat{\mathbf{x}} \in P$. Therefore $\lim x_{[n]}^{0}\left(t_{2}\right) \rightarrow \hat{x}^{0}\left(t_{2}\right)$. By $(22), \hat{x}^{0}\left(t_{2}\right)=M$. Q.E.D.

Theorem 7. If there is an allowed path which terminates at $\xi_{2}$ at some $t_{2} \leqq T_{2}$, there is an optimal control among the alluwed set which terminates at $\xi_{2}$ on or before $T_{2}$. $\left(S_{0}\left(T_{2}\right)\right.$ is not empty.)

Proof. Let $\lambda$ denote the straight line

$$
\boldsymbol{x}=\boldsymbol{\xi}_{2}
$$

in the $(n+1)$-dimensional $x$-space. A point on $\lambda$, is specified by its value of $x^{0}$. Let

$$
I=\lambda \cap \Omega\left(t \leqq T_{2}\right)
$$

Each point on $I$ is the terminal point of an allowed path and vice versa. $I$ is not empty because of the condition of the theorem.

As $I$ is the intersection of a closed set $\lambda(\lambda$ includes $\pm \infty)$, and a closed set 
$\Omega\left(t \leqq T_{2}\right)$ (Theorem 5), $I$ is closed. Therefore, there is a point $\hat{\mathbf{x}} \in I$ such that

$$
\hat{\mathbf{x}}^{0}=\sup _{x \in I} x^{0} .
$$

Since $\hat{\mathbf{x}} \in I \subset \Omega\left(t \leqq T_{2}\right)$, there is at least one allowed path leading to $\hat{\mathbf{x}}$ on or within $T_{2}$. Q.E.D.

COROLlary. If there is an allowed path terminating at $\xi_{2}$, in finite time $\left(x\left(t_{2}\right)=\xi_{2}\right)$, there is a minimal time control among the allowed set terminating at $\xi_{2}$.

Proof. Since there is no need to consider any allowed path which terminates at $\xi_{2}$ at a later instant, $T_{2}=t_{2}$. The corollary is reduced to a special case of Theorem 7 with $f^{0}(x, u, t)=-1$.

V. Optimum control in bounded phase space.

Definitions and assumptions.

Definitions. Subscripts are used to denote components of covariant vectors:

$$
\begin{aligned}
& \psi=\left(\psi_{0}, \psi\right)=\left(\psi_{0}, \psi_{1}, \psi_{2}, \cdots, \psi_{n_{1}}\right), \\
& \eta=\left(\eta_{0}, \eta\right)=\left(\eta_{0}, \eta_{1}, \eta_{2}, \cdots, \eta_{n_{1}}\right) .
\end{aligned}
$$

(Note. A light face greek letter $\psi$ or $\eta$ without subscript is meant to be the enlarged vector $\left(\psi_{0}, \psi\right)$ or $\left(\eta_{0}, \eta\right)$. A light face greek letter with a subscript is meant to be a component of the vector.)

The concept of "magnitude" of a vector does not enter into the problem nor the theorems. However, in proving the theorems, it is desirable to have these concepts so that bounds can be defined or calculated. The magnitude of a vector $x$ is denoted as $|x|$ and defined by

$$
|x|^{2} \equiv\left(x^{1}\right)^{2}+\left(x^{2}\right)^{2}+\cdots+\left(x^{n}\right)^{2} .
$$

The same is true for covariant vectors. In the subsequent sections, "distance" is redefined in terms of the magnitude rather than the norm.

Assumptions. The region $X$ and functions $f^{i}(x, u, t)$ are assumed to satisfy the following conditions:

5.1. $X$ is $n$-dimensional.

5.2. A unique normal exists on every bounded point $x_{b}$ of $X$.

The unit vector in the direction of the normal at $x_{b}$ pointing outward is designated by $\eta\left(x_{b}\right)$. The component $\eta_{0}$ is identically zero.

5.3. The partial derivatives $\partial \eta_{i}(x) / \partial x^{j}$ are uniformly bounded.

5.4. Every point $x$ within a certain distance $d_{i}$ from $X$ is on one and only one of the normals $\eta\left(x_{b}\right)$. The normal $\eta\left(x_{b}\right)$ therefore can also be identified as $\eta(x)$, where $x$ may be any point on $\eta$ within a distance $d_{i}$ from the boundary. 
5.5. There exists a distance $d_{2}, d_{1}>d_{2}>0$, and a constant $a_{i}>0$, such that at every point $x$ within distance $d_{i}$ from $X$ there are at least some $u \in U$ satisfying:

$$
\sum_{i=1}^{i=n} \eta_{i}(x) \cdot f^{i}(x, u, t)<-a_{1}, \quad u \in U .
$$

The closed region extending outward from the boundary of $X$ up to distance $d_{2}$ is denoted by $X_{0}$.

$$
X+X_{0} \subset X_{1}
$$

$A$ constructed problem. Because of assumption 5.4, a distance function $v(x)$ can be defined as follows:

$$
\begin{aligned}
& v(x)=0 \quad \text { if } x \in X, \\
& v(x)=\sum_{i=1}^{i=n} \eta_{i}(x)\left(x^{i}-x_{b}^{i}\right) \quad \text { if } \quad x \in X_{0} .
\end{aligned}
$$

Let the problem defined in $\$ I I I$ with the added assumptions be denoted as $P$. A constructed problem $P(K)$ is defined as follows:

5.6. In (8), the equation for $i=0$ is replaced by

$$
\dot{x}^{0}=f^{0}(x, u, t)-K[v(x)]^{2} .
$$

5.7. Equation (11) is replaced by

$$
x(t) \in X+X_{0}, \quad t_{1} \leqq t \leqq t_{2} .
$$

The allowed controls satisfy conditions $3.1,3.2,3.3$. The terminal condition is that $t_{2}$ is fixed but $\boldsymbol{x}\left(t_{2}\right)$ is free.

VI. Optimal path of the constructed problem. An allowed path can be generated by choosing any $u(t)$ when $x(t)$ is an interior point of $X+X_{0}$, and choosing a $u(t)$ which gives

$$
\sum_{i=1}^{i=n} \eta_{i}(x) f^{i}(x, u, t)<0
$$

when $x(t)$ is a boundary point of $X+X_{0}$. From Theorem 6 , the optimum control exists.

Lemma 4. Given any distance $d, 0<d<d_{2}$, a sufficiently large $M(d)$ can be found such that if $K>M(d), v(x)<d$ for every point $x$ on an optimal path $\Gamma_{0}(K)$ of $P(K)$.

Proof. Since $\mathrm{f}$ is bounded there is a constant $B_{1}$ such that

$$
|\mathbf{f}(\boldsymbol{x}, \boldsymbol{u}, t)|<B_{1}
$$


for all $x, u$, and $t$ in the product region $\left(X+X_{0}\right) U T$. Then

$$
\frac{d}{d t} v(x)=\sum_{i}\left[\dot{\eta}_{i}(x)\left(x^{i}-x_{b}^{i}\right)-\eta_{i} \dot{x}_{b}^{i}+\eta_{i} f^{i}\right]=\sum_{i} \eta_{i} f^{i}
$$

The first and second terms vanish since $\dot{\eta}$ and $\dot{x_{b}}$ are perpendicular to the normal but $x-x_{b}$ is parallel to the normal. Therefore

$$
\left|\frac{d}{d t} v(\hat{x})\right| \leqq B_{1}
$$

Let the largest value of $v(x)$ be denoted by $d_{3}$. Since $x\left(t_{1}\right) \in X, t_{2}-t_{1}$ $\geqq d_{3} / B_{1}$. Then

$$
\int_{t_{1}}^{t_{2}}[v(\hat{x})]^{2} d t \geqq \frac{1}{3} \frac{d_{3}^{3}}{B_{1}}
$$

Let $R_{1}$ represent the return of any allowed path of $P$. The same path is also an allowed path in problem $P(K)$ with the same return. Let $R_{0}(K)$ denote the return of the optimal $\Gamma_{0}(K)$, then

But

$$
R_{0}(K) \geqq R_{1} \text {. }
$$

$$
R_{0}(K) \leqq B_{1}\left(t_{2}-t_{1}\right)-\frac{K}{3} \frac{d_{3}^{3}}{B_{1}}
$$

Therefore

$$
\frac{K}{3} \frac{d_{3}^{3}}{B_{1}} \leqq B_{1}\left(t_{2}-t_{1}\right)-R_{1}
$$

Let

$$
M(d) \equiv \frac{3 B_{1}\left[B_{1}\left(t_{2}-t_{1}\right)-R_{1}\right]}{d^{3}}
$$

If

$$
K>M(d)
$$

then

$$
\frac{K}{3} \frac{d_{3}^{3}}{B_{1}}>\frac{d_{3}^{3}}{d^{3}}\left[B_{1}\left(t_{2}-t_{2}\right)-R_{1}\right]
$$

Since $B_{1}\left(t_{2}-t_{1}\right)-R_{1}$ is always positive, (31) and (32) give $d_{3}<d$. Q.E.D.

In the subsequent paragraph, some properties on the displacements about a given path are derived. It is assumed that $x \in X+X_{0}$ but $K=0$. Thus the calculated $\mathrm{x}$ is $\mathrm{x}(t, 0)$. The result can be applied to a problem with $K \neq 0$ by modifying only $\delta x^{0}$ :

$$
\delta x^{0}(t, K)=\delta x^{U}(t, 0)-K \int_{t_{1}}^{t} \delta[v(x)]^{2} d t .
$$


Let $\epsilon$ denote an infinitesimal quantity, and $\Delta x, \Delta t$, etc., variations of the order unity and are independent of $\epsilon$. Following an initial displacement $\delta \mathbf{x}\left(t_{3}\right)=\epsilon \Delta \mathbf{x}\left(t_{3}\right)+\cdots$, the subsequent change in $\mathbf{x}(t)$ can be expressed as

$$
\delta \mathbf{x}(t)=\epsilon \Delta \mathbf{x}(t)+\cdots
$$

where $\cdots$ means infinitesimal quantities of higher order in $\epsilon$. From (8)

$$
\Delta \dot{x}^{i}(t)=\sum_{j=1}^{j=n_{1}}\left(\frac{\partial f^{i}}{\partial x^{j}}\right)_{\Gamma} \Delta x^{j}(t)
$$

where the subscript $\Gamma$ means that the partial derivatives are evaluated along the original path.

Lemma 5. Let $K=0$, and $x(t) \in X+X_{0}$. At any point $x\left(t_{3}\right) \in X_{0}$ along an allowed path with

$$
\frac{d}{d t} v(x) \geqq-\frac{a_{1}}{3},
$$

it is possible to apply a $u_{1} \in U$ for an infinitesimal interval $\epsilon \Delta t$ to cause a subsequent displacement $\epsilon \Delta \mathbf{x}$ from the original path satisfying

$$
-\sum_{i=1}^{i=n_{1}} \eta_{i}(x(t)) \Delta x^{i}(t)>M_{1} \Delta t, \quad t_{3}<t \leqq t_{3}+\tau
$$

and

$$
|\Delta \mathbf{x}|<M_{2} \Delta t, \quad t_{3} \leqq t \leqq t_{2},
$$

where $M_{1}, M_{2}$, and $\tau$ are constants independent of $x$.

Proof. From (30)

$$
\sum_{i=1}^{i=n_{1}} \eta_{i}\left(x\left(t_{3}\right)\right) f^{i}\left(x\left(t_{3}\right), u, t_{3}\right) \geqq-\frac{a_{1}}{3} .
$$

From assumption 5.5, there is a $u_{1}$ satisfying (24). Applying $u_{1}$ instead of $u$ for an infinitesimal interval gives

$$
\Delta x^{t}\left(t_{3}\right)=\left[f^{\prime}\left(x\left(t_{3}\right), u_{1}, t_{3}\right)-f^{\prime}\left(x\left(t_{3}\right), u, t_{3}\right)\right] \Delta t .
$$

From (24) and (38) one obtains

$$
\sum_{i=1}^{i=n} \eta_{i}\left(x\left(t_{3}\right)\right) \Delta x^{i}\left(t_{3}\right)<-\frac{2}{3} a_{1} \Delta t
$$

From (35)

$$
\frac{d}{d t} \sum_{i=1}^{i=n}\left[\Delta x^{i}\right]^{2}=2 \sum_{i, j=1}^{i, j=n}\left(\frac{\partial f^{i}}{\partial x^{j}}\right)_{\mathrm{r}} \Delta x^{i} \Delta x^{J}
$$


Let $2 \lambda$ denote the upper bound of the eigenvalue of the symmetrical matrix with

$$
\left(\frac{\partial f^{i}}{\partial x^{j}}+\frac{\partial f^{\prime}}{\partial x^{i}}\right)_{r}
$$

as its $i, j$ th element. (An upper bound exists because $\partial f^{i} / \partial x^{j}$ are bounded for all $i$ and $j$.) Then for any given $t$, the right-hand side of (40) is shown to be bounded by $2 \lambda|\Delta x(t)|^{2}$ by a linear transform of $\Delta x(t)$. It follows then

$$
\frac{d}{d t}|\Delta \mathbf{x}(t)| \leqq \lambda|\Delta \mathbf{x}(t)| .
$$

The solution is

$$
|\Delta \mathbf{x}(t)| \leqq e^{\lambda\left(t-t_{3}\right)}\left|\Delta \mathbf{x}\left(t_{3}\right)\right|, \quad t>t_{3} .
$$

Since

$$
\left|\Delta \mathbf{x}\left(t_{3}\right)\right| \leqq 2 B_{1} \Delta t,
$$

one has

$$
|\Delta x(t)| \leqq 2 B_{1} e^{\lambda\left(t-t_{i j}\right)} \Delta t .
$$

Inequality (37) is satisfied by choosing an $M_{2}$ as follows:

$$
\begin{array}{rlrl}
M_{2} & =2 B_{1} e^{\lambda\left(t_{2}-t_{1}\right)} & & \text { if } \lambda>0, \\
& =2 B_{1} & & \text { if } \lambda \leqq 0 ; \\
\frac{d}{d} \sum_{i=1}^{i=n} \eta_{i} \Delta x^{i} & =\sum_{i} \sum_{j} \frac{\partial \eta_{i}}{\partial x^{j}} f^{j} \Delta x^{i}+\sum_{i} \sum_{j} \eta_{i} \frac{\partial f^{i}}{\partial x^{j}} \Delta x^{j} \\
& =\sum_{i} \sum_{j}\left[f^{j} \frac{\partial \eta_{i}}{\partial x^{j}}+\eta_{j} \frac{\partial f^{j}}{\partial x^{i}}\right] \Delta x^{i} .
\end{array}
$$

The sum

$$
\sum_{j}\left[f^{\prime} \frac{\partial \eta_{i}}{\partial x^{j}}+\eta_{j} \frac{\partial f^{\prime}}{\partial x^{i}}\right]
$$

is the $i$ th component of a covariant vector. Let $\alpha$ denote the vector. There is a $B_{2}>|\alpha|$ on $\left(X+X_{0}\right) U T$, since $f^{\prime}, \eta_{j}, \partial f^{\prime} / \partial x^{l}$, and $\partial \eta_{i} / \partial x^{j}$ are all bounded. Equation (42) gives

$$
\frac{d}{d} t \sum_{i=1}^{i=n} \eta_{i} \Delta x^{i}<B_{2}|\Delta x|<B_{2} M_{2} \Delta t .
$$

Let $M_{1}=a_{1} / 3$, and $\tau=a_{1} / 3 B_{2} M_{2}$, inequality (36) is satisfied. Q.E.D. Lemma 6. Let $\Gamma_{0}(K)$ be the optimal path in problem $P(K)$. For sufficiently 
large $K$, it is possible to find a sequence of $t$ of no more than $2\left(t_{1}, t_{2}\right) / \tau$ members: $t_{1}^{\prime}, t_{2}^{\prime}, \cdots, t_{m}^{\prime}, \cdots, t_{N}^{\prime}$ such that all the following conditions are satisfied:

(i) $t_{1} \leqq t_{1}^{\prime}<t_{2}^{\prime} \cdots t_{N}^{\prime}<t_{2}, N \leqq 2\left(t_{2}-t_{1}\right) / \tau$.

(ii) Along $\Gamma_{0}(K)$

$$
\sum_{i=1}^{i=n_{1}} \eta_{i}\left(\hat{x}\left(t_{m}^{\prime}\right)\right) f^{i}\left(\hat{x}\left(t_{m}^{\prime}\right), \hat{u}, t_{m}^{\prime}\right)>-\frac{a_{1}}{3}, \quad m=1,2, \cdots, N .
$$

(iii) Along $\Gamma_{0}(K), v(\hat{x}(t))=0$ unless $t$ is in one of the intervals $t_{m}^{\prime} \leqq \mathrm{t} \leqq t_{m}^{\prime}+\tau$.

Proof. Choose a $K$ sufficiently large such that along an optimal path $\Gamma_{0}(K)$

$$
v(\hat{x})<\frac{a_{1} \tau}{6} .
$$

The time interval $t_{1}<t<t_{2}$ is divided into subintervals of equal duration $\tau / 2$. Let the subintervals be numbered in chronological order. If, in a subinterval, there are some values of $t$ such that

$$
\hat{\boldsymbol{x}}(t) \in X_{0}+\text { boundary of } X
$$

and (43) is satisfied, then the smallest $t$ which satisfies (43) and (45) is selected as one of the $t_{m}^{\prime}$. If (43) and (45) are not simultaneously satisfied for any $t$ in a subinterval, no $t_{m}^{\prime}$ will be selected. The $t_{m}^{\prime \prime} \mathrm{s}$ are then numbered in chronological order.

The total number of $t_{m}^{\prime}$ is no more than the total number of subintervals, and (i) and (ii) are automatically satisfied.

If there is a $t_{m}^{\prime}$ in the $k$ th subinterval, condition (iii) is satisfied in the $(k+1)$ st subinterval. If there is no $t_{m}^{\prime}$ in the $k$ th subinterval, either $\hat{\boldsymbol{x}}$ is in the interior of $X$ for the entire $k$ th subinterval, or $\hat{x}$ is on the boundary or outside of $X$ for some part of the subinterval, but $v(\hat{x})$ is decreasing at a faster rate than what is permitted by (43). In the latter case, since $\hat{x}$ is continuous in $t, v(\hat{x})$ must be monotonically decreasing in $t$ for as long as $v(\hat{x}) \in X_{0}$. Since (44) is satisfied at the beginning of the period, $\hat{x}$ must be in the interior of $X$ at the end of the $k$ th subinterval. In any case $\hat{x}$ at the beginning of the $(k+1)$ st subinterval must be a point in $X$. Since in leaving $X,(43)$ and (45) are always satisfied, and the smallest $t$ in the $(k+1)$ st subinterval satisfying (43) and (45), (if any), is chosen as a $t_{m}^{\prime}$, (iii) is again satisfied for the $(k+1)$ st subinterval.

Since $k$ can be any value from 1 to $N-1$, and $\hat{x}\left(t_{1}\right) \in X$, (iii) is satisfied for the entire interval $t_{1} \leqq t \leqq t_{2}$. Q.E.D.

Lemma 7. For sufficiently large $K$, it is possible to find a $M_{3}$ independent of $K$ such that

$$
K \int_{t_{1}}^{t_{2}} v(\hat{x}) d t<M_{3}
$$


where $\hat{x}(t)$ describes an optimal path of $P(K)$.

Proof. Inequality (46) can be proved by induction. Suppose

$$
K \int_{t_{m+1}}^{t_{2}} v(\hat{x}) d t<C_{m+1},
$$

where $C_{m+1}$ is a constant independent of $K$. Following a change in $u$ at $t_{m}^{\prime}$, there is a $\delta \mathbf{x}\left(t_{m}^{\prime}\right)=\epsilon \Delta \mathbf{x}\left(t_{m}^{\prime}\right)$. At $t=t_{2}$

$$
\begin{aligned}
\Delta x^{0}\left(t_{2}, K\right)= & \Delta x^{0}\left(t_{2}, 0\right)-\int_{t_{m}}^{t_{2}} 2 K v(\hat{x}) \sum_{i=1}^{i=n} \frac{\partial v(x)}{\partial x^{i}} \Delta x^{i} d t \\
= & \Delta x^{0}\left(t_{2}, 0\right)-2 K \int_{t_{m}^{\prime}}^{t_{2}} v(\hat{x}) \sum_{i} \eta_{i} \Delta x^{i} d t, \\
\Delta x^{0}\left(t_{2}, K\right)= & \Delta x^{0}\left(t_{2}, 0\right)-2 K \int_{t_{m+1}^{\prime}}^{t_{2}} v(\hat{x}) \sum_{i} \eta_{i} \Delta x^{i} d t \\
& -2 K \int_{t_{m}^{\prime}}^{t^{\prime}} v(\hat{x}) \sum_{i} \eta_{i} \Delta x^{i} d t .
\end{aligned}
$$

Since $\hat{x}(t)$ is an optimal path, $\Delta x^{0}\left(t_{2}, K\right) \leqq 0$. Because of Lemma 6 , it is possible for $v(\hat{x})$ to differ from zero in the interval $t_{m}^{\prime} \leqq t \leqq t_{m+1}^{\prime}$ only if $t<t_{m}^{\prime}+\tau$. Substituting (36), (37), and (47), into (48) gives

$$
\begin{aligned}
& 2 K \int_{t_{m}}^{t^{\prime}} v(\hat{x}) d t \cdot M_{1} \Delta t<M_{2} \Delta t+2 C_{m+1} M_{2} \Delta t, \\
& K \int_{t_{m}^{\prime}}^{t_{m+1}^{\prime}} v(\hat{x}) d t<\frac{M_{2}}{M_{1}}\left(\frac{1}{2}+C_{m+1}\right), \\
& K \int_{t_{m}^{\prime}}^{t_{2}} v(\hat{x}) d t<\frac{M_{2}}{M_{1}}\left(\frac{1}{2}+C_{m+1}\right)+C_{m+1} \equiv C_{m} .
\end{aligned}
$$

Let $t_{N+1}^{\prime}=t_{2}$. Inequality (47) is obviously true for $m=N$. Repeating (49) $N$ times gives (46). Q.E.D.

Choose $K$ sufficiently large so that $d<d_{2}$. The optimal path is in the interior of $X+X_{0}$.

Let $\hat{\mathrm{u}}(t)$ and $\hat{\mathbf{x}}(t, K)$ denote an optimal control and optimal path pair for problem $P(K)$. Due to a change of $u(t)$ for an infinitesimal period $\epsilon \Delta t$ at $t-$.

$$
\begin{aligned}
\epsilon \Delta \mathbf{x}(t, K) & =\mathbf{x}(t, K)-\hat{\mathbf{x}}(t, K)+\cdots \\
& =[\mathbf{f}(\hat{\mathbf{x}}, \boldsymbol{u}, t)-\mathrm{f}(\hat{x}, \hat{u}, t)] \epsilon \Delta t .
\end{aligned}
$$

The significances of $\epsilon$ and $\Delta x, \Delta t$ are stated in the proof of Lemma 5 . From $(35), \mathbf{x}\left(t_{2}, K\right)$ is related to $\Delta \mathbf{x}(t, K)$ by a linear transform: 


$$
\Delta x^{i}\left(t_{2}, K\right)=\sum_{j=0}^{j=n_{1}} A_{j}^{i}\left(t_{2}, t\right) \Delta x^{J}(t, K), \quad i=0,1,2, \cdots, n_{1} .
$$

Since $\hat{x}(t, K)$ is an optimal path

$$
\Delta x^{0}\left(t_{2}, K\right) \leqq 0 .
$$

Let the covariant vector $A_{j}^{0}\left(t_{2}, t\right)$ be denoted as $\psi_{j}(t)$. The above inequality can be written as

$$
\sum_{i=0}^{i=n_{1}} \psi_{i}(t)\left[f^{\prime}(\hat{x}, u, t)-f^{\prime}(\hat{x}, \hat{u}, t)\right] \leqq 0 .
$$

Let the Hamiltonian function be defined as

$$
H(\psi, x, u, t) \equiv \sum_{i=0}^{i=n_{1}} \psi_{i}(t) f^{i}(x, u, t)
$$

Inequality (52) implies

$$
H(\psi, \hat{x}, \hat{u}, t)=\sup _{u \in U} H(\psi, \hat{x}, u, t) .
$$

For problem $P(K)$, equation (35) is modified by adding $-K[v(x)]^{2}$ to $f^{0}(x, u, t)$. With the modified (35), it can be readily shown that

$$
\dot{\psi}_{i}+\sum_{k=0}^{k=n} \dot{\psi}_{k} \frac{\partial f^{k}(x, u, t)}{\partial x^{i}}=2 \psi_{0} K v(x) \frac{\partial v(x)}{\partial x^{i}}
$$

By definition of $v(x)$ :

$$
\begin{aligned}
\frac{\partial v(x)}{\partial x^{i}} & =\eta_{i}(x) & & \text { if } x \in X_{0}, \\
& =0 & & \text { if } x \in X .
\end{aligned}
$$

Substituting (56), into (55) gives

$$
\psi_{i}+\sum_{j} \psi_{j} \frac{\partial f^{j}(x, u, t)}{\partial x^{i}}=2 \psi_{0} K v(x) \eta_{i}(x), \quad i=0,1,2, \cdots, n_{1} .
$$

A boundary condition for $\psi_{i}(t)$ is

$$
\begin{aligned}
\psi_{i}\left(t_{2}\right) \equiv A_{i}^{0}\left(t_{2}, t_{2}\right)=0 & \text { if } i \neq 0, \\
=1 & \text { if } i=0 .
\end{aligned}
$$

Since $f^{\prime}(u, x, t)$ is independent of $x^{u}$, and $\eta_{0}(x)=0$, the equation for $i=0$ in (57) gives $\psi_{0}=0$. Therefore

$$
\psi_{0}(t)=1, \quad t_{1} \leqq t \leqq t_{2} .
$$


The above shows that along a path $\Gamma_{0}(K)$ which maximizes the integral

$$
R(K)=\int_{t_{1}}^{t_{2}}\left\{f^{0}(x, u, t)-K[v(x)]^{2}\right\} d t
$$

the control $u(t)$ maximizes the Hamiltonian function at every point.

VII. In the limit of $K \rightarrow \infty$. In the subsequent proof, the variables associated with each $\Gamma_{0}(K)$ need to be clearly designated. The symbols $\psi(t, K)$, $\mathbf{x}(t, K), u(t, K)$, etc., will be used instead of $\psi(t), \mathbf{x}(t), u(t)$. Whenever confusion is not possible, the short forms $\mathrm{x}, u$ may be used with $(t, K)$ understood. The capped functions will be reserved for the limit functions whenever they exist as $K \rightarrow \infty$.

DEFinitions. The following functions are defined along an optimal path $\Gamma_{0}(K)$ of $\mathrm{P}(K)$ :

$$
\begin{aligned}
J(t, K) & \equiv K \int_{t}^{t_{2}} v(x) \eta(x) d t, \\
G_{j}(t, K) & \equiv \int_{t}^{t_{2}} \sum_{i} \psi_{i}(t, K)\left(\frac{\partial f^{i}}{\partial x^{j}}\right)_{\mathrm{r}_{0}(K)} d t .
\end{aligned}
$$

Lemma 8. The functions $J_{i}(t, K)$ has uniformly bounded variations on $T$ for all $K, i=1,2, \cdots, n_{1}$.

Proof. Choose any ordered set $t_{3}, t_{4}, \cdots, t_{2 N}$ such that $t_{1} \leqq t_{3} \leqq t_{4} \leqq \cdots$ $\leqq t_{2 n} \leqq \cdots \leqq t_{2}$. Then

$$
\begin{aligned}
\sum_{n=2}^{n=N} \mid J_{i}\left(t_{2 n-1}, K\right) & -J_{i}\left(t_{2 n}, K\right) \mid \\
& =\sum_{n=2}^{n=N} K\left|\int_{t_{2 n-1}}^{t_{2 n}} v(x) \eta_{i}(x) d t\right| \leqq \sum_{n=2}^{n=N} K \int_{t_{2 n-1}}^{t_{2 n}} v(x) d t<M_{3} .
\end{aligned}
$$

The last inequality sign follows from (46). Q.E.D.

It follows from Theorem 1 and Lemma 8 that $\left\{J_{i}(t, K)\right\}$ are equimeasurable.

Lemma 9. The functions $\psi(t, K)$ of $\Gamma_{0}(K)$ are uniformly bounded (independent of $K$ ).

Proof. Since $\psi\left(t_{2}, K\right)$ is known, (57) is solved backwards. The homogeneous equation $(K=0)$ gives for $t_{3}<t$ :

$$
\psi_{i}\left(t_{3}, K\right)=\sum_{j=0}^{j=n_{1}} \psi_{j}(t, K) A_{i}^{\jmath}\left(t, t_{3}\right) .
$$

By superposition, the solution of the nonhomogeneous equation is, for $i \neq 0$, 


$$
\psi_{i}\left(t_{3}, K\right)=-\sum_{j=1}^{j=n_{1}} \int_{t_{3}}^{t_{2}} A_{i}^{j}\left(t, t_{3}\right) 2 K v(x) \eta_{j}(x) d t
$$

for any given $i, A_{i}^{j}\left(t, t_{3}\right)$ is the $j$ th component of a contravariant vector $A_{i}^{j}\left(t, t_{3}\right)$. By differentiating (63), and eliminating $\psi$ with the aid of (57), $A_{i}^{j}\left(t, t_{3}\right)$ is shown to satisfy (35). Using the same steps which lead to (41) one obtains

$$
\left|\mathbf{A}_{i}\left(t, t_{3}\right)\right|<B_{4}, \quad i=0,1, \cdots, n_{1},
$$

where $B_{4} \equiv \sup \left\{1, e^{\lambda\left(t_{2}-t_{1}\right)}\right\}$. Thus (64) gives

$$
\left|\psi_{i}\left(t_{3}, K\right)\right| \leqq \int_{t_{3}}^{t_{2}}\left|\mathrm{~A}_{i}\left(t, t_{3}\right)\right| 2 K v(x) d t<2 B_{4} M_{3} \equiv B_{5} .
$$

Q.E.D.

Lemma 10. The set of functions $\mathrm{G}(t, K)$ are equicontinuous.

Lemma 10 follows from the fact the integrand on the right-hand side of (62) is uniformly bounded.

Lemma 11. The set of functions $\psi(t, K)$ for all $\Gamma_{0}(K)$ is equimeasurable.

Proof. Integrating (57) with respect to $t$ from $t$ to $t_{2}$ gives

$$
\psi_{i}\left(t_{2}\right)-\psi_{i}(t, K)+G_{i}(t, K)=J_{i}(t, K) .
$$

Since equicontinuous functions are equimeasurable, and the sum or difference of equimeasurable functions are equimeasurable, Lemma 11 follows. Q.E.D.

Let $\boldsymbol{w}_{(k)}(t)$ denote a vector function with components $u(t, K), \mathbf{x}(t, K)$, $\mathrm{G}(t, K), \mathrm{J}(t, K), \psi(t, K)$. Let $A_{i}$ denote a set of $\left\{w_{(K)}(t)\right\}$ with $K=K_{0}$, $K_{0}+1, \cdots, \infty$; where $K_{0}$ is sufficiently large $K$ so that $d<d_{2}$ is satisfied for the optimal path $\Gamma_{0}(K)$. Corollary 2 of Theorem 2 states that there is a subsequence $w_{|K|}(t) \in A_{i}$ such that $w_{|K|}(t)$ converges to a limit uniformly or almost uniformly in all its components

$$
\begin{gathered}
u(t, \mathrm{~K}) \stackrel{[a . u .]}{\longrightarrow} \hat{u}(t), \\
\mathbf{x}(t, \mathrm{~K}) \stackrel{[u]}{\longrightarrow} \hat{\mathbf{x}}(t), \\
\mathrm{G}(t, \mathrm{~K}) \stackrel{[u]}{\longrightarrow} \hat{\mathrm{G}}(t), \\
\mathrm{J}(t, \mathrm{~K}) \stackrel{[\text { a.u.] }}{\longrightarrow} \hat{\mathrm{J}}(t), \\
\psi(t, \mathrm{~K}) \stackrel{[\text { a.u. }]}{\longrightarrow} \hat{\psi}(t),
\end{gathered}
$$

where $\mathrm{K}$ means a selected set satisfying $w_{|\mathrm{K}|}(t) \in\left\{w_{(\mathrm{K})}(t)\right\}$. 
Lemma 12. The function $\hat{\mathbf{x}}(t) \in X$ for every $t$ on $T$.

Proof. Since $X$ is closed, the distance

$$
d\left(\hat{x}\left(t_{3}\right), X\right)>a>0
$$

for some $t_{3}$ if Lemma 12 is not true.

Lemma 4 states that there is a finite $M(a / 2)$ so that for every $\mathrm{K}>M(a / 2)$

$$
d\left(\boldsymbol{x}\left(t_{3}, \mathrm{~K}\right), X\right)<a / 2
$$

and (68) implies

$$
\left\|\hat{x}\left(t_{3}\right)-\boldsymbol{x}\left(t_{3}, \mathrm{~K}\right)\right\|<a / 2
$$

for every $K$ greater than some finite $K(a / 2)$. Thus (72) is contradicted by choosing a $K$ sufficiently large.

Lemma 13. The limit $\hat{a} \in C$ and $\hat{a}$ and $\hat{\mathbf{x}}$ form an allowed control and path pair.

The proof is identical with that of Theorem 3 .

Extensions of $\hat{J}(t), \hat{\psi}(t)$. Equation (70) implies that $J(t, K)$ converges to $\hat{J}(t)$ on a set $T-T^{\prime}$, where $T^{\prime}$ is a subset of $T$ having zero measure. Since $\hat{J}(t)$ is defined only as a limit of $\mathrm{J}(t, K)$, it is not defined on $T^{\prime}$.

Since $T^{\prime}$ is a set of no measure, and $T$ is a closed interval, every point of $T^{\prime}$ is a limit point of $T-T^{\prime}$. Lemma 8 and (70) assures $\hat{J}(t)$ to be of bounded variation. Therefore, one sided limit exists for $\hat{\jmath}$ at each point of $T^{\prime}$.

Definition. For each point $t \in T^{\prime}, \hat{J}(t)$ is defined to be

$$
\hat{J}(t) \equiv \lim _{\epsilon \rightarrow 0} \mathrm{~J}(t+|\epsilon|), \quad t+|\epsilon| \in T-T^{\prime}
$$

with the above definition, $\mathrm{J}(t)$ is completely defined on $T$.

In the limit of $K \rightarrow \infty$, (66) converges to

$$
\psi\left(t_{2}\right)-\hat{\psi}(t)+\hat{\mathrm{G}}(t)=\hat{J}(t)
$$

except on a set of zero measure. One may define a $\tilde{\psi}(t)$ such that

$$
\psi\left(t_{2}\right)-\tilde{\psi}(t)+\hat{\mathrm{G}}(t)=\hat{J}(t)
$$

holds everywhere. Since $\tilde{\psi}(t)$ and $\hat{\psi}(t)$ are different only on a set of zero measure, (71) holds for $\widetilde{\psi}(t)$ also. One then discards $\hat{\psi}(t)$ and redesignates $\widetilde{\psi}(t)$ as $\hat{\psi}(t)$. Consequently (73) holds everywhere.

Lemma 14. The function $\hat{\jmath}(t)$ can be expressed as

$$
\begin{aligned}
\hat{J}(t) & =\int_{t}^{t_{2}} \zeta\left(t^{\prime}\right) \eta\left(\hat{x}\left(t^{\prime}\right)\right) d t^{\prime}, \\
\zeta(t) & =0 \text { if } x \text { is an interior point of } X, \\
& \geqq 0 \text { if } x \text { is a boundary point of } X .
\end{aligned}
$$


Proof. Let $t_{3}$ and $t_{4}$ be any two points on $T-T^{\prime}$ with $t_{4}>t_{3}$. Then by definition (61)

$$
\begin{aligned}
\hat{\jmath}\left(t_{3}\right)-\mathfrak{\jmath}\left(t_{4}\right) & =\lim _{K \rightarrow \infty}\left[J\left(t_{3}, K\right)-J\left(t_{4}, K\right)\right] \\
& =\lim _{K \rightarrow \infty} \int_{t_{3}}^{t_{4}} K v(x(t, K)) \eta(x(t, K)) d t .
\end{aligned}
$$

Let

$$
\mathcal{J}\left(t_{3}, t_{4}\right) \equiv \hat{\jmath}\left(t_{3}\right)-\hat{\jmath}\left(t_{4}\right)
$$

$\jmath\left(t_{3}, t_{4}\right)$ can be separated into two integrals:

$$
\jmath\left(t_{3}, t_{4}\right)=\mathrm{J}_{1}\left(t_{3}, t_{4}\right)+\mathrm{J}_{2}\left(t_{3}, t_{4}\right),
$$

where

$$
\mathrm{J}_{1}\left(t_{3}, t_{4}\right)=\lim _{K \rightarrow \infty} \int_{t_{3}}^{t_{4}} K v(x(t, K)) \eta(\hat{x}(t)) d t
$$

and

$$
\mathrm{J}_{2}\left(t_{3}, t_{4}\right)=\lim _{K \rightarrow \infty} \int_{t_{3}}^{t_{4}} K_{v}(x(t, K))[\eta(x(t, K))-\eta(\hat{x}(t))] d t .
$$

Since $\partial \eta_{i} / \partial x^{j}$ are bounded for all $i, j, \eta(x)$ satisfies a Lipschitz condition:

$$
\begin{aligned}
& \left|\int_{t_{3}}^{t_{4}} K v(x(t, K))[\eta(x(t, K))-\eta(\hat{x}(t))] d t\right| \\
& \leqq M_{3} B_{\eta} \sup \|(x(t, K))-\hat{x}(t)\|, \quad t_{3} \leqq t \leqq t_{4} .
\end{aligned}
$$

Therefore $J_{2}\left(t_{3}, t_{4}\right)=0$ and

$$
\jmath\left(t_{3}, t_{4}\right)=\mathrm{J}_{1}\left(t_{3}, t_{4}\right) .
$$

Let $J(K)$ denote

$$
\int_{t_{3}}^{t_{4}} K v(x(t, K)) \eta(\hat{x}(t)) d t .
$$

The interval $\left[t_{3}, t_{4}\right]$ is partitioned into segments of no greater than $\tau$. Let these intervals be denoted $\tau_{i}$, and $\hat{x}_{i}$ denote an arbitrary point of $\hat{x}(t)$ with $t \in \tau_{i}$.

Then

$$
\left|J(K)-\sum_{i} \eta\left(\hat{x}_{i}\right) \int_{\tau_{i}} K v(x(t, K)) d t\right| \leqq M_{3} B_{\eta}\left\|\hat{x}(t)-\hat{x}_{i}\right\| \leqq M_{3} B_{\eta} B_{1} \tau .
$$

In the limit of $K \rightarrow \infty$, (78) becomes

$$
\left|\mathrm{J}_{1}\left(t_{3}, t_{4}\right)-\sum_{i} \eta\left(\hat{\boldsymbol{x}}_{i}\right) \zeta_{i} \tau_{i}\right|<M_{3} B_{\eta} B_{1} \tau,
$$


where $\zeta_{i}$ is some unknown quantity. It is zero if $x$ is an interior point of $X$ and nonnegative because $K_{v}(x(t, K))$ is non-negative.

In the limit of $\tau \rightarrow 0$, (79) becomes

$$
\mathrm{J}_{1}\left(t_{3}, t_{4}\right)=\int_{t_{3}}^{t_{4}} \zeta(t) \eta(\hat{x}) d t .
$$

Equations (77) and (80) are equivalent to (74). Inequality (75) follows from the condition on $\zeta_{i}$. Q.E.D.

From (67), (68), (69), and (71) it is obtained

$$
\hat{G}_{j}(t)=\int_{t_{3}}^{t_{4}} \frac{\partial}{\partial \hat{x}_{j}} H\left(\hat{\psi}, \hat{x}, \hat{u}, t^{\prime}\right) d t^{\prime},
$$

where the function $H$ is defined in (53).

LEMMa 15.

$$
\sum_{i} \hat{\psi}_{i} f^{i}(\hat{x}, \hat{u}, t)=\sup _{u \in U} \sum_{i} \hat{\psi}_{i} f^{i}(\hat{x}, u, t)
$$

for $t \in T-T^{\prime}$, where $\mu\left\{T^{\prime}\right\}=0$.

Proof. From (67) and (71), $u(t, K)$ and $\psi(t, K)$ converge uniformly to $\hat{u}(t)$ and $\hat{\psi}(t)$ except on a set $T^{\prime}$ of zero measure.

Note that $U$ is closed. If (82) is not true then there is a $u_{1}(t)$ such that $t \in T-T^{\prime}$ and

$$
\sum_{i} \hat{\psi}_{i} f^{i}\left(\hat{x}, \boldsymbol{u}_{1}, t\right)-\sum_{i} \hat{\psi}_{i} f^{i}(\hat{x}, \hat{u}, t)>a>0
$$

a contradiction can be shown as follows.

As $\mathrm{f}$ is continuous in $\boldsymbol{x}$ and $u$, a sufficiently large $K$ can be found such that

$$
\left|\sum_{i} \hat{\psi}_{i} f^{i}\left(\hat{x}, u_{1}(t), t\right)-\sum_{i} \psi_{i}(t, K) f^{i}\left(x(t, K), u_{1}(t), t\right)\right|<\frac{a}{2}
$$

and

$$
\left|\sum_{i} \psi_{i}(t, K) f^{i}(\boldsymbol{x}(t, K), \boldsymbol{u}(t, K), t)-\sum_{i} \hat{\psi}_{i} f^{i}(\hat{x}, \hat{u}, t)\right|<\frac{a}{2} .
$$

The above inequalities and (83) give

$$
\sum_{i} \psi_{i}(t, K) f^{i}\left(x(t, K) u_{1}(t), t\right)>\sum_{i} \psi_{i}(t, K) f^{i}(x(t, K), u(t, K), t) .
$$

But (84) contradicts (54) (the reader is reminded the change of notation explained at the beginning of the present section), therefore $u_{1}(t)$ cannot exist. Q.E.D.

It follows from (82) and the definition (53) that 


$$
H(\hat{\psi}, \hat{x}, \hat{u}, t)=\sup _{u \in U} H(\hat{\psi}, \hat{x}, u, t)
$$

almost everywhere on $T$. The set $T^{\prime}$ on which (85) does not hold has zero measure.

Let $\tilde{u}(t)$ be defined as $\hat{u}(t)$ on $T-T^{\prime}$ and one of the $u^{\prime}$ 's satisfying (85) for every $t$ on $T^{\prime}$. Then the Hamiltonian is maximized by $\tilde{u}(t)$ everywhere. Since $\mu\left\{T^{\prime}\right\}=0$, all the previously derived relations for $\hat{u}(t) \quad((67)$, Lemma 13 , and (81)) are equally valid for $\tilde{u}(t)$. They are so replaced, and $\tilde{u}$ is redenoted $\hat{u}$. Thus (85) holds everywhere for the new $\hat{u}$.

Substituting (80) and (81) into (73) and differentiating the resulting equation gives

$$
\frac{\partial \hat{\psi}_{j}}{\partial t}+\frac{\partial H(\hat{\psi}, \hat{x}, \hat{u}, t)}{\partial \hat{x}^{j}}=\zeta(t) \eta_{j}(\hat{x}(t)) .
$$

VIII. Theorem 8 and Corollary. The results of the preceding sections can be summarized into a theorem:

TheOREm 8. For at least one $\hat{u}(t)$ among the set of optimal controls with free end point in a problem defined by equation (8) and conditions (3.1) to (3.3), and (5.1) to (5.5) there exist $\zeta(t)$ and $\hat{\psi}(t)$ such that $\hat{u}(t), \hat{\mathbf{x}}(t), \zeta(t)$, and $\hat{\psi}(t)$ satisfy equations (58), (75), (85), and (86).

Definition. An allowed control $\hat{u}(t)$ is said to be an isolated locally optimal control if for every allowed $\hat{u}_{1}(t)$ with $R\left(u_{1}(t)\right) \geqq R(\hat{u}(t))$ a constant $b>0$ and a time $t_{3}, t_{1} \leqq t_{3} \leqq t_{2}$ can be found such that

$$
\left\|x_{1}\left(t_{3}\right)-\hat{x}\left(t_{3}\right)\right\|>b,
$$

where $\hat{x}(t)$ is the path resulting from $\hat{u}(t)$.

Corollary. For every isolated locally optimal control $\hat{u}(t)$ with free end point in a problem defined by equation (8) and conditions (3.1) to (3.3), (5.1) to (5.5), there exists a function $\zeta(t)$ and a function $\psi(t)$ such that $\hat{u}(t)$, $\hat{x}(t), \zeta(t)$ and $\psi(t)$ satisfy equations (58), (75), (85), and (86).

Proof. A function $F(x, t)$ is defined by

$$
\begin{array}{ll}
F(x, t)=0 & \text { if }|x-\hat{x}(t)|<b / 2, \\
F(x, t)=(|x-\hat{x}(t)|-b / 2)^{2} & \text { otherwise. }
\end{array}
$$

The function $\hat{x}(t)$ is treated as a given function of $t$. It is readily verified that $F(x, t)$ is bounded in the accessible region in $x$-space, has continuous and bounded derivatives in $x$, and is continuous in $t$. A constructed problem is defined by adding $-K_{1} F(x, t)$ to the $f^{0}(x, u, t)$. With sufficiently large $K_{1}, \hat{u}(t)$ becomes the only optimal control in the constructed problem, and the Corollary follows from Theorem 8. Q.E.D. 
IX. Proof that the necessary condition is also sufficient for linear systems with convex allowed region $X$. The controlled system is called linear if

$$
f^{i}(x, u, t) \equiv \sum_{j=1}^{j=n_{1}} F_{j}^{i}(t) x^{j}+\sum_{k=1}^{k=n_{2}} B_{k}^{i}(t) u^{k}+C^{i}(t) .
$$

Let the set $\hat{u}(t), \hat{\mathbf{x}}(t)$, together with $\zeta(t)$, and $\hat{\psi}(t)$ satisfy equations (8), (58), (75), (85), and (86). It is readily shown from equations (8), (86), and (88) that for any other allowed set $u(t), \mathbf{x}(t)$

$$
\begin{aligned}
\frac{d}{d t} \sum_{i=0}^{i=n_{1}} \hat{\psi}_{i}(t)\left[\hat{x}^{i}(t)-x^{i}(t)\right]= & \sum_{i} \zeta(t) \eta_{i}(x)\left[\hat{x}^{i}(t)-x^{i}(t)\right] \\
& +\sum_{i} \sum_{k} \hat{\psi}_{i}(t) B_{k}^{i}(t)\left[\hat{u}^{k}(t)-u^{k}(t)\right] .
\end{aligned}
$$

Integrating (89) from $t_{1}$ to $t_{2}$ and making use of (58)

$$
\begin{aligned}
R(\hat{u})-R(\boldsymbol{u})= & \int_{t_{1}}^{t_{2}} \zeta(t) \sum_{i} \eta_{i}(\hat{x})\left[\hat{x}^{i}-x^{i}\right] d t \\
& +\int_{t_{1}}^{t_{2}} \sum_{i} \sum_{k} \hat{\psi}_{i}(t) B_{k}^{i}(t)\left[\hat{u}^{k}-u^{k}\right] d t
\end{aligned}
$$

Due to the convexity of $X$, and (75)

$$
\zeta(t) \sum_{i} \eta_{i}(\hat{x})\left[\hat{x}^{i}-x^{i}\right] \geqq 0 .
$$

Due to (85)

$$
\sum_{i} \sum_{k} \hat{\psi}_{i}(t) B_{k}^{i}(t)\left[\hat{u}^{k}-u^{k}\right] \geqq 0
$$

Therefore

$$
R(\hat{u})-R(\boldsymbol{u}) \geqq 0 .
$$

Acknowledgement. The work upon which this paper was based was sponsored by the Office of Scientific Research, Air Research and Development Command, Washington, D. C., under Grants No. AF-AFOSR-62-321 and AF-AFOSR-542-64.

\section{REFERENCES}

1. R. V. Gamkrelidze, Optimal control processes with restricted phase coordinates, Izv. Akad. Nauk SSSR Ser. Mat. 24 (1960), 315-356. Translated by L. W. Neustadt, Space Technology Laboratories Report, March, 1961.

2. S. S. L. Chang, Minimal time control with multiple saturation limits, IEEE Trans. Automatic Control AC-8 (1963), 35-42.

3. V. G. Boltjanskii,, R. V. Gamkrelidze, and L. S. Pontryagin, The theory of optimal 
processes. I. Maximum principle, Izv. Akad. Nauk SSSR Ser. Mat. 24 (1960), no. 1. Translated by L. W. Neustadt, Space Technology Laboratories, Report No. 9810. 32-01, October, 1960.

4. R. V. Gamkrelidze, The theory of time optimal process in linear systems, Izv. Akad. Nauk SSSR 2 (1958), 449-474. Translated by members of the staffs of the University of California and Space Technology Laboratories, January, 1961.

5. E. B. Lee and L. Markus, On the existence of optimal controls, Preprint ASME-61-JAC-2, Amer. Soc. Mechanical Engineers, Chicago, Ill.,

6. L. M. Graves, The theory of functions of real variables, McGraw-Hill, New York, 1956; Theorem 23, p. 66 .

7. M. E. Munroe, Introduction to measure and integration, Addison-Wesley, Reading, Mass., 1959, p. 225.

8. - Introduction to measure and integration, Addison-Wesley, Reading, Mass., 1959 ; p. 222.

9. L. M. Graves, The theory of functions of real variables, McGraw-Hill, New York, 1956; Theorem 2, p. 100 and Theorem 25, p. 121.

10. - The theory of functions of real variables, McGraw-Hill, New York, 1956; Theorem 23, p. 66.

State University of New York at Stony Brook, STONY BROOK, L. I., NEW YoRK 